\title{
Efeitos da oclusão temporal na geração de opções na tomada de decisão de atletas e não atletas de voleibol
}

http://dx.doi.org/10.11606/1807-5509201900010099

\begin{tabular}{|c|c|}
\hline $\begin{array}{r}\text { Henrique de Oliveira CASTRO* } \\
\text { Gustavo De Conti Teixeira COSTA } \\
\text { Gibson Moreira PRAÇA*/** } \\
\text { Flávio de Oliveira PIRES } \\
\text { Pablo Juan GRECO* }\end{array}$ & $\begin{array}{l}\text { *Escola de Educação } \\
\text { Fisica, Fisioterapia e } \\
\text { Terapia Ocupacional, } \\
\text { Universidade Federal } \\
\text { de Minas Gerais, Belo } \\
\text { Horizonte, MG, Brasil. } \\
\text { **Departamento de } \\
\text { Educação Fisica, } \\
\text { Universidade } \\
\text { Federal dos Vales } \\
\text { do Jequitinhonha e } \\
\text { Mucuri, Diamantina, } \\
\text { MG, Brasil. } \\
\text { ***Departamento de } \\
\text { Educação Fisica, } \\
\text { Universidade Federal } \\
\text { do Maranhão, São } \\
\text { Luís, MA, Brasil. }\end{array}$ \\
\hline
\end{tabular}

\section{Resumo}

Objetiva-se investigar o efeito da oclusão temporal na quantidade de opções geradas no momento da tomada de decisão (TD) de atletas e não atletas de voleibol. Participaram 46 voluntários, sendo 23 atletas e 23 não atletas. Para análise da quantidade de opções geradas no momento da TD, utilizou-se o Teste de Conhecimento Tático Declarativo no Voleibol (TCTD:Vb) realizado em duas situações: com e sem oclusão temporal. Quando comparados os dois grupos na situação com oclusão, observa-se que os atletas geraram mais opções do que os não atletas. Quando comparadas as duas situações, geraram-se mais opções na situação sem oclusão do que na com oclusão nos dois grupos. Conclui-se que a quantidade de opções geradas depende das estratégias utilizadas para a resolução de uma ação e do tempo disponível para visualizar determinada situação de jogo.

Palavras-Chave: Voleibol; Cognição; Tomada de decisão; Geração de opções.

\section{Introdução}

A otimização do conhecimento tático ${ }^{1}$, das capacidades perceptivo-visuais e da tomada de decisão (TD) são imprescindíveis em esportes abertos como o voleibol, caracterizados por situaçóes e ambiente variável ${ }^{2}$, movimentos rápidos da bola, focalizaçóes alternadas entre oponente e ambiente ${ }^{3,4}$, pressão de tempo e diversas opções para resolução da ação $0^{5-7}$.

O processo de TD em uma situação real de jogo envolve a análise de diversas variáveis que se interagem e estáo em constante mudança ${ }^{6,8,9}$, sendo que processos cognitivos como percepção ${ }^{10-12} \mathrm{e}$ atenção ${ }^{13-17}$ são condicionantes da TD. Além disso, ela sofre influências de fatores como o número de alternativas, compatibilidade de estímulo-resposta ${ }^{18}$ de acordo com a complexidade da situação e condicionantes de pressão temporal, espacial, motora, entre outras, impostos na jogada ${ }^{6}$.

Um dos focos de estudo da TD direciona-se ao entendimento das escolhas realizadas pelo participante dentro das várias opçóes apresentadas durante uma situação de jogo ${ }^{19-21}$. Na pesquisa científica na área das Ciências do Esporte, considera-se que a quantidade de opçóes geradas está relacionada com o comportamento criativo do sujeito para a $\mathrm{TD}^{22-25}$.

Em estudos que utilizam cenas de vídeo para analisar a TD nos esportes, um dos procedimentos utilizados é a oclusão temporal, a qual tem como objetivo investigar possíveis decréscimos na qualidade das opçóes geradas, o que permite compreender quanto o participante consegue extrair e processar as informaçóes necessárias 
ao longo da exibição da sequência do filme, caracterizando um desempenho expert. Oclusão temporal em cenas de vídeo relaciona-se com o corte da cena em determinado momento da ação. Além disso, situaçóes experimentais de oclusão temporal induzem processos intuitivos de TD, ou seja, pressupóem maior utilização da memória de trabalho na representação da situação, o que influencia na subsequente geração de opçóes e na qualidade dessas soluçóes ${ }^{17,22,26,27}$.

Contrário a isso, quando se recorre a cenas de vídeo nas que a TD se realiza sem oclusão temporal, a exposição da cena congelada durante a fase de geração de opçóes garante uma condição de percepçáo constante, na qual a primeira opção a ser gerada pelo participante será produzida sob a mesma condição em relação à última opção, favorecendo assim os processos deliberativos de $\mathrm{TD}^{21,22}$.

Um dos componentes essenciais a ser desenvolvido nos jogos esportivos coletivos e influencia diretamente na TD é o conhecimento tático. Ele reflete a competência do participante de planejar com antecedência e influenciar as decisóes dentro de uma situação ${ }^{7}$. Nos esportes, os peritos com grande conhecimento tático específico na modalidade conseguem gerar um número menor de opçóes com maior qualidade ${ }^{28} \mathrm{e}$ obtêm respostas mais rápidas ${ }^{21}$ do que os novatos na $\operatorname{TD}^{17,29}$.

Observa-se uma lacuna na literatura envolvendo tarefas e açóes específicas de modalidades esportivas utilizando-se de situaçóes de oclusão temporal, principalmente na modalidade voleibol. Devido às características da modalidade voleibol, que nas açóes específicas de jogo (AE, AC, LE e BL) o tempo é curto, faz-se necessário o entendimento do efeito causado pela oclusáo temporal, visto que a quantidade de opçóes para a resolução da tarefa demonstra uma gama de opçóes para escolha e consequente sucesso da ação. Desta forma, o presente estudo objetiva investigar o efeito da oclusão temporal na quantidade de opçóes geradas no momento da tomada de decisão (TD) de atletas e náo atletas de voleibol nas ações de ataque de extremidade (AE), ataque de centro (AC), levantamento (LE) e bloqueio (BL).

\section{Método}

Participaram 46 voluntários do sexo masculino divididos em dois grupos: G1) atletas ( $\mathrm{n}=23$; idade média de 16,9 \pm 1 anos; experiência com treinamento sistematizado de voleibol de $3,7 \pm 1,1$ anos; treino 5 vezes por semana; duraçáo média de cada sessáo de treino de 90 minutos; disputam campeonatos a nível regional, estadual e nacional), e G2) não atletas ( $\mathrm{n}=23$; idade média de 17,6 $\pm 1,7$ anos e nenhuma experiência com treinamento sistematizado de voleibol).

O projeto foi aprovado pelo Comitê de Ética em Pesquisa (Parecer 971.037) e todos os voluntários, bem como seus representantes legais, concederam consentimento por escrito para participação no estudo.

As coletas dos dados com os atletas ocorreram nos próprios clubes e com os não atletas em um laboratório. Em ambos os espaços, o teste aplicouse em uma sala propícia para a realização dos procedimentos da pesquisa. Todos os testes foram agendados previamente com cada voluntário, que foram conduzidos até o local de realização, no qual estava presente apenas o pesquisador responsável. Os participantes sentavam-se confortavelmente em uma cadeira de frente para o computador no qual decorreriam as cenas de vídeo que pertencem ao teste.

Utilizou-se o Teste de Conhecimento Tático Declarativo no Voleibol (TCTD: $\mathrm{Vb})^{30}$, que consiste em 24 cenas reais de jogos de voleibol apresentadas em quatro tipos de açóes: $\mathrm{AE}$ (seis cenas), AC (seis cenas), LE (seis cenas) e BL (seis cenas). As cenas exibidas duram de quatro a seis segundos, todas elas filmadas na perspectiva de topo, com distância aproximada de sete a nove metros da quadra, permitindo ao observador a visão total da quadra e percepção de profundidade nas diferentes situaçóes.

As açóes de $\mathrm{AE}$ e $\mathrm{AC}$ iniciam-se a partir do saque adversário, perpassam pela recepçáo da equipe do outro lado da rede, pelo levantamento e interrompe-se a imagem no momento do ataque. Para as situaçóes de $\mathrm{AE}$, as opçóes esperadas eram: ataque na diagonal, ataque na paralela, largada ou explorar o bloqueio. Para as situações de AC, as opçóes esperadas eram: ataque na posição 1 , ataque na posiçáo 5, ataque frontal, largada ou explorar o bloqueio. 
As açóes de LE e BL iniciam-se a partir do saque, perpassam pela recepção e interrompem-se no momento do levantamento. Para as situações de LE, as opçóes esperadas eram: levantar para a posição 4, levantar para a posiçáo 3, levantar para a posição 2 , levantar para a posição 1 ou levantar para a posição 6. Para as situaçóes de BL, as opçóes esperadas eram: bloquear na posição 2 , bloquear na posição 3 ou bloquear na posição 4 .

Antes de iniciar os testes, de forma padronizada, realizou-se os processos de instruçóes relativas ao teste, além de uma adaptação ao procedimento experimental, que se repetiu em ambas as situaçóes (com e sem oclusão). A adaptação consistiu em visualizar duas cenas de cada ação (AE, AC, BL e LE) com durações de quatro a seis segundos, seguindo exatamente os mesmos protocolos da situação experimental. Terminada a adaptação e sem questionamentos ou dúvidas, por parte do voluntário, iniciavam-se os testes experimentais. Reitera-se que os testes foram realizados em duas situaçôes: com oclusão temporal (situação 1) e sem oclusão temporal (situaçáo 2). A ordem de realizaçáo dos testes foi escolha dos pesquisadores.

$\mathrm{Na}$ situação 1 (com oclusão temporal), objetiva-se extrair as informações relacionadas ao comportamento visual que leva a TD aproximandose o teste ao máximo do momento temporal em uma situação de jogo real. Bordini et al. ${ }^{31}$ citam que nos estudos que utilizam a oclusão temporal, uma sequência de ação (vídeo) é paralisada em determinado ponto de sua execução na tentativa de identificar períodos de tempo dentro dos quais atletas experientes conseguem identificar o resultado final da ação baseado em dicas visuais relevantes. Assim, no momento da interrupção da cena, a tela se apaga e o voluntário verbaliza imediatamente o que fazer (primeira opção - TD). Em seguida, visando outras possíveis alternativas de solução da jogada que o voluntário tenha percebido na cena, consideram-se até 22 segundos para responder verbalmente "o que mais?" $17,20,26,29,31,32$, explicitando-se a fase de geração de opções, além de responder por último qual a melhor das opçóes dentre as alternativas produzidas. Com este procedimento é possível relacionar aspectos como a geração de opçôes, criatividade, qualidade e tipo da TD: intuitiva ou deliberativa ${ }^{5}$.

$\mathrm{Na}$ situação 2 (sem oclusão temporal), na interrupção da cena, congela-se a imagem na tela por três segundos (a cena permanece na tela na forma de uma fotografia) e nesse tempo o voluntário responde "o que fazer?", que se refere à sua TD (primeira opção gerada). Em seguida, o voluntário dispóes de até 22 segundos para responder "o que mais?" 17,19-21,26,31,32, verbalizando outras possíveis alternativas de soluçâo da jogada (geração de opçóes).

Objetiva-se com as duas situaçôes verificar a influência da pressão de tempo em relação a diferenças na percepção de sinais relevantes, na geração de opçóes e na qualidade da TD dos voluntários. $\mathrm{Ou}$ seja, se o tempo para a análise da cena repercute na quantidade de opçóes geradas para tomar as decisóes, onde compara-se a cena dinâmica (com oclusão) e a estática (sem oclusão).

Para a descrição das respostas dos voluntários em cada situação apresentada utilizou-se uma ficha de controle, seu preenchimento manual ficou a cargo do pesquisador responsável durante a realização dos testes. Assim, analisaram-se a geração de opçôes, que se refere à possíveis alternativas de solução da jogada que o voluntário tenha percebido na cena, bem como a qualidade da TD produzidas para cada ação $0^{33-35}$.

Para minimizar o efeito da memória na aplicação das situaçóes de coleta, cada voluntário realizou primeiro o teste com oclusão e após 48 horas ${ }^{5}$, o mesmo voluntário realizou o teste sem oclusão com a ordem de apresentação modificada e as cenas aleatorizadas, como descritas abaixo no QUADRO 1.

Observa-se no QUADRO 1 que o teste na situação com oclusão (situação 1) apresentou-se para os voluntários seguindo a ordem de açóes de jogo: $\mathrm{AE}, \mathrm{AC}, \mathrm{LE}$ e BL. Na situação sem oclusão (situação 2) seguiu-se outra ordem de açóes: LE, AE, BL e AC. A ordem de apresentação das cenas em cada situação e ação também se modificou, conforme descrito no QUADRO 1.

$\mathrm{Na}$ análise dos dados, recorreu-se à análise de variância de dois fatores (ANOVA two-way), para os fatores grupo (2 níveis - atletas e não atletas) e situaçóes (2 níveis - com e sem oclusão). Para as variáveis nas quais detectou-se interação entre fatores principais, realizou-se ainda uma análise de desdobramentos. O post hoc de LSD realizou-se para múltiplas comparações. Para todas as análises utilizou-se o software SPSS 20.0, mantendo-se o nível de significância em 5\%. 
$\mathrm{AE}=$ Ataque de Extremidade;

$\mathrm{AC}=$ Ataque de Central;

$\mathrm{LE}=$ Levantamento; $\mathrm{BL}=$ Bloqueio.

QUADRO 1 - Apresentação do teste nas situações com e sem oclusão visual

\begin{tabular}{|l|c|c|}
\hline Situaçáo & Ordem Das Situaçóes & Ordem Das Cenas \\
\hline \multirow{3}{*}{ Com oclusão visual } & $\mathrm{AE}$ & $13 \mathrm{~A}, 33 \mathrm{~A}, 48 \mathrm{~A}, 67 \mathrm{~A}, 81 \mathrm{~A}, 86 \mathrm{AA}$ \\
\cline { 2 - 3 } & $\mathrm{AC}$ & $62 \mathrm{~A}, 64 \mathrm{~A}, 84 \mathrm{~A}, 117 \mathrm{~A}, 141 \mathrm{~A}, 214 \mathrm{~A}$ \\
\cline { 2 - 3 } & $\mathrm{LE}$ & $43 \mathrm{~L}, 53 \mathrm{~L}, 54 \mathrm{~L}, 89 \mathrm{~L}, 91 \mathrm{~L}, 101 \mathrm{~L}$ \\
\cline { 2 - 3 } & $\mathrm{BL}$ & $1 \mathrm{~B}, 4 \mathrm{~B}, 14 \mathrm{~B}, 16 \mathrm{~B}, 17 \mathrm{~B}, 56 \mathrm{~B}$ \\
\hline \multirow{3}{*}{ Sem oclusão visual } & $\mathrm{LE}$ & $89 \mathrm{~L}, 91 \mathrm{~L}, 53 \mathrm{~L}, 54 \mathrm{~L}, 43 \mathrm{~L}, 101 \mathrm{~L}$ \\
\cline { 2 - 3 } & $\mathrm{AE}$ & $14 \mathrm{~A}, 17 \mathrm{~B}, 1 \mathrm{~B}, 56 \mathrm{~A}, 48 \mathrm{~A}, 13 \mathrm{~A}, 67 \mathrm{~A}, 16 \mathrm{~B}$ \\
\cline { 2 - 3 } & $\mathrm{BL}$ & $64 \mathrm{~A}, 84 \mathrm{~A}, 141 \mathrm{~A}, 117 \mathrm{~A}, 62 \mathrm{~A}, 214 \mathrm{~A}$ \\
\cline { 2 - 3 } & $\mathrm{AC}$ & \\
\cline { 2 - 3 } & &
\end{tabular}

\section{Resultados}

A TABELA 1 abaixo descreve as médias e desvio-padrão das análises da quantidade de opções geradas entre os grupos nas duas situaçôes em cada ação apresentada.

De acordo com os resultados apresentados na TABELA 1 na situação com oclusão, o grupo de atletas apresentou maior média do que o grupo de não atletas $(\mathrm{p}=0,001)$. Observa-se também que o grupo atletas apresentou maior média na situação com oclusão quando comparada à situação sem oclusão $(\mathrm{p}=0,002)$.

Nas ações AC e BL, na situação com oclusão o grupo de atletas apresentou maior média do que o grupo de não atletas nas duas açóes - AC e BL ( $\mathrm{p}=0,04$ e $\mathrm{p}=0,001$, respectivamente). $\mathrm{Na}$ comparação entre os grupos, o de não atletas apresentou maior média na situação sem oclusão quando comparada à situação com oclusão nas duas açôes $-\mathrm{AC}$ e BL ( $\mathrm{p}=0,04$ e $\mathrm{p}=0,001$, respectivamente).

$\mathrm{Na}$ ação LE o grupo de atletas demonstrou maiores valores médios nas duas situações em comparação ao grupo de não atletas. Além disso, a situação sem oclusão apresentou maiores valores médios do que a situação com oclusão nos dois grupos.
$\mathrm{AE}=$ Ataque de extremidade; $\mathrm{AC}=$ Ataque de centro; $\mathrm{BL}=$ Bloqueio; $\mathrm{LE}=$ Levantamento;

*: atletas $>$ não atletas na situação com oclusão $(p=0,001)$; $\square$ com oclusão > sem oclusão no grupo de atletas $(p=0,002)$ : ** atletas $>$ não atletas na situação com oclusão $(p=0,04) ;{ }^{\circ}:$ sem oclusão > com oclusão no grupo de não atletas $(p=0,04) ; * *$ : atletas $>$ não atletas na situação com oclusão $(p=0,001) ;$; sem oclusão > com oclusão no grupo de não atletas $(p=0,001) ; * * * *$ : atletas > não atletas nas duas situações $(p=0,001) ;:$ Sem oclusão > com oclusão nos dois grupos $(p=0,001)$.

TABELA 1 - Média ( \pm desvio-padrão) da quantidade de opções geradas entre os grupos em cada ação e cada situação

\begin{tabular}{|c|c|c|c|}
\hline & & Atletas & Náo Atletas \\
\hline \multirow{2}{*}{$\mathbf{A E}$} & Com Oclusão & $2,25( \pm 0,72)^{*} \mathrm{f}$ & $1,78( \pm 0,78)$ \\
\hline & Sem Oclusão & $1,92( \pm 0,61)$ & $1,90( \pm 0,74)$ \\
\hline \multirow{2}{*}{$\mathrm{AC}$} & Com Oclusão & $1,83( \pm 0,75)^{* *}$ & $1,65( \pm 0,76)$ \\
\hline & Sem Oclusão & $1,75( \pm 0,70)$ & $1,83( \pm 0,66)^{\circ}$ \\
\hline \multirow{2}{*}{ BL } & Com Oclusão & $1,91( \pm 0,55)^{* * *}$ & $1,34( \pm 0,62)$ \\
\hline & Sem Oclusão & $1,87( \pm 0,52)$ & $1,78( \pm 0,68) \S$ \\
\hline \multirow{2}{*}{ LE } & Com Oclusão & $2,05( \pm 0,67)^{* * * *}$ & $1,75( \pm 0,74)$ \\
\hline & Sem Oclusão & $2,11( \pm 0,62) 2$ & $1,81( \pm 0,70)$ \\
\hline
\end{tabular}

\section{Discussão}

Neste estudo verificou-se o efeito da oclusão temporal na quantidade de opçóes geradas de atletas e não atletas de voleibol no momento da TD nas açóes de AE, AC, LE e BL.
Em relação às situações com e sem oclusão temporal, conforme os resultados encontrados, observou-se uma diferença significativa no número de opçóes geradas, que foram maiores para o grupo 
de atletas quando comparados com o grupo de não atletas na situação com oclusão temporal em todas as açōes de jogo. As estratégias de busca de sinais relevantes nos momentos com e sem oclusão evidenciaram-se como um importante fator que comprova as diferenças encontradas nos resultados deste estudo, afirmando a hipótese proposta por JOHNSON e RAAB ${ }^{19}$, cujo os autores citam que a diferença entre o número e os tipos de opçóes geradas depende das estratégias utilizadas para a resolução de uma determinada ação.

CAUSER e Ford ${ }^{26}$ realizaram uma investigação com 205 estudantes de uma pós graduação em Ciências do Esporte (idade média de $20 \pm 0,8$ anos; 155 homens e 55 mulheres) divididos em: a) jogadores de futebol $(n=106)$, b) de outros esportes de invasão $(\mathrm{n}=43)$ e c) de outros esportes $(\mathrm{n}=58)$. Os pesquisadores utilizaram cenas de vídeos de jogos de futebol $4 \times 4$ com oclusão temporal na TD para a resolução das cenas (analisando-se a quantidade de opçóes geradas e a qualidade da primeira opção). Como utilizado no presente estudo na situação com oclusão, a cena desaparecia decorridos quatro segundos, tempo esse utilizado para a tomada de decisão. A hipótese de que a exatidão da TD ocorre entre esportes de características semelhantes foi confirmada, não sendo encontradas diferenças entre os grupos estudados. Porém, os autores citam que em esportes com características diferentes dos jogos de invasão, como o voleibol, não se confirmou essa hipótese. No presente estudo, no voleibol, verificou-se que a precisão das respostas varia de acordo com o nível de experiência na modalidade e tempo disponível para análise da situação de jogo apresentada.

Bordini et al. ${ }^{31}$ analisaram o efeito da oclusão temporal na cortada do voleibol sobre a tomada de decisão defensiva em atletas com diferentes níveis de experiência. Participaram 48 atletas de voleibol divididos em três grupos caracterizados por categorias e níveis. Os autores utilizaram diferentes momentos de corte caracterizando os momentos de oclusão temporal e avaliaram a qualidade da TD dos atletas. Uma das tarefas consistia em informar o local de aterrisagem da bola. A oclusão temporal utilizada no presente estudo nas situaçóes de $\mathrm{AE}$ e $\mathrm{AC}$ representam o exato momento do contato entre $\mathrm{o}$ atacante e a bola, apresentado por BoRDINI et al. ${ }^{31}$ como OT4, sendo essa situação a de maior frequência de acertos dentre todas as outras de pré-contato. A diferença principal entre o presente estudo e o realizado por Bordini et al. ${ }^{31}$ emerge da análise da quantidade de opções geradas no momento com oclusão temporal e também na comparaçáo com o momento sem oclusão, impossibilitando assim a comparação dos resultados encontrados.

Em relação aos grupos, no presente estudo encontraram-se diferenças significativas no que tange as geraçóes de opçóes dos grupos nas situações de jogo apresentadas. $\mathrm{Na}$ situação de $\mathrm{AE}$, o grupo de atletas gerou mais opçóes quando ocorreu a oclusão temporal do que na sem oclusão. Nas situaçóes de AC e BL, o grupo de não atletas gerou mais opçóes na situação sem oclusão do que na situação com oclusão.

Particularmente na situaçáo de LE, encontraramse diferenças significativas no número de opçóes geradas sendo que o grupo de atletas gerou mais opções do que o grupo náo atleta nos dois momentos. Além disso, no momento sem oclusão gerou-se mais opçôes do que no momento com oclusão nos dois grupos.

Em um estudo realizado no futebol por Memmert et al. ${ }^{33}$ com 30 jogadores com média de idade de 25,27 $( \pm 3,72)$ anos e experiência de 17,93 $( \pm 4,87)$ anos, utilizaram-se 20 cenas de vídeo de jogos de situaçóes ofensivas com aproximadamente dez segundos de duração. Após a interrupção da cena, o voluntário tinha até um minuto para gerar opçóes de respostas para solucionar a situação apresentada. Os participantes foram avaliados quanto aos critérios de criatividade das respostas: originalidade, flexibilidade e fluência ${ }^{36-38}$. Os autores encontraram diferenças significativas nas condições emocionais para os três critérios de criatividade das respostas, concluindo que a geraçáo de soluçóes criativas pode ser otimizada por meio da mudança no estado motivacional dos atletas. No presente estudo, não se avaliou a criatividade das opçóes geradas e o estado motivacional dos participantes, impossibilitando a comparação dos resultados obtidos.

Utilizando-se do congelamento de cenas dinâmicas (sem oclusáo temporal), JoHnson e RAAB $^{19}$ realizaram um estudo com 85 jogadores masculinos de handebol com média de idade de 15,6 ( $\pm 1,54)$ anos do Brasil e Alemanha. As cenas tinham uma duraçáo aproximada de dez segundos e após a parada, congelavam-se as imagens durante 45 segundos, tempo esse para a TD e geraçáo de opções. Os atletas obtiveram uma média de geração de 2,3 opçôes nas situaçôes de jogo apresentadas. Os autores apresentam ainda que não houve uma correlaçáo entre a média do número de opçóes geradas e a qualidade da melhor opçáo escolhida. 
Ainda de acordo com Johnson e RAAB ${ }^{19}$, uma hipótese do efeito da geração de opçóes na TD radica em que a diferença entre o número de opçóes geradas depende das estratégias utilizadas pelo voluntário, isto é, quando este define um tipo de estratégia necessária para a resolução de uma determinada ação, gera diferentes tipos e quantidades de opçóes. Fato esse que pode ser explicado pelo conhecimento tático e experiência específica da modalidade e situaçóes vivenciadas, retomadas da memória ${ }^{35,19-42}$, que resulta nas diferenças emergentes das estratégias utilizadas. Essas estratégias se evidenciam nos estudos que envolvem o rastreamento ocular, nas modalidades esportivas coletivas em que o ambiente varia, como o caso do voleibol, assim, comprova-se que diferenças nas quantidades, duraçóes e locais de fixaçóes visuais são deterministas para uma TD correta ${ }^{17,31,43-45}$.

No estudo de Belling et al. ${ }^{34}$, utilizando uma tarefa de futebol, objetivou-se investigar o tempo de restrição sobre o comportamento de geração de opçôes durante uma fase de avaliação e intervenção na TD. Participaram 21 jogadores de futebol de nível recreacional (idade média de 19,8 $\pm 1,94$ anos; experiência média na modalidade de 10,19 $\pm 6,04$ anos), que avaliaram 30 videoclipes de aproximadamente 5 a 10 segundos de duração em situaçôes normais de jogo (11x11). Utilizou-se a oclusão temporal da cena em um determinado momento para a TD dos participantes. Os resultados fornecem um apoio adicional no comportamento de geração de opçóes consistente com a proposta de Johnson e RAAB ${ }^{19}$ denominada de heurística do "Take-the-Fist" durante a fase de intervenção sem restrição de tempo.

O trabalho no futebol realizado por BeLLING et al. ${ }^{35}$ teve como um dos objetivos testar mecanismos cognitivos propostos para explicar o desempenho dos especialistas na TD, observou-se que durante a fase de intervenção a heurística do "Take-the-First" proposta por JoHNSON e RAAB ${ }^{19}$ foi prevalente.
Os resultados das investigaçóes realizados por BeLling et al. ${ }^{34,35}$ corroboram com os encontrados no presente estudo e confirmam a hipótese proposta por JOHNSON e RAAB ${ }^{19}$, visto que as estratégias visuais utilizadas para as análises das cenas independente das situaçōes apresentadas diferiram entre os atletas e não atletas no número e duração das fixaçóes visuais. A qualidade da TD e a quantidade de opçóes geradas também foram influenciadas pelas estratégias de busca visual diferentes entre atletas e não atletas.

O treinamento específico dos processos cognitivos (percepção, atenção, TD) por meio da organização dos processos de E-A-T relacionados ao conhecimento de sinais relevantes dentro da periodização técnicotática, torna-se relevante para o desenvolvimento do conhecimento tático, facilitando a realização das ações motoras de forma mais eficiente por parte do atleta, integrando-se no conceito de proceduralização do conhecimento conforme postulado por ANDERSON ${ }^{46}$. Além disso, os processos de E-A-T aliados a diferentes formas de jogos, aumentam a vivência prática da modalidade, assim armazenam-se na memória informaçóes que são utilizadas nas interações dos processos de bottom-up e top-down ${ }^{6}$, resultando em uma melhor e mais rápida TD.

Conclui-se com os resultados encontrados no presente estudo que a quantidade de opçóes geradas depende das estratégias utilizadas para a resolução de uma ação e do tempo disponível para visualizar determinada situação de jogo. A quantidade de opções geradas também é influenciada pela criatividade do atleta, sendo que atletas mais criativos geram mais opções para solução de situaçóes de jogo. Sugere-se a investigação dos efeitos de diferentes modelos de treinamento técnico-táticos e processos de E-A-T que objetivem a melhora da percepção, atenção e TD nas diferentes situaçóes de jogo do voleibol, e, com isso, consequente melhora no conhecimento tático específico da modalidade, criatividade e aumento de opções geradas para solucionar determinada ação.

\section{Abstract}

Effects of temporal occlusion on the options generation of decision-making in volleyball athletes and non-athletes

Objective is investigate the effect of temporal occlusion on the number of options generated in the decision-making (DM) moment of athletes and non-athletes of volleyball. There were 46 participants, 23 athletes and 23 non-athletes. For the analysis of the number of options generated at the TD moment, the Declarative Tactical Knowledge Test in Volleyball (DTKT:Vb) as used in two situations: with and without temporal occlusion. When comparing the two groups in the situation with occlusion, it is observed that the 
athletes generated more options than the non-athletes. When comparing the two situations, more options were generated in the without occlusion than in the occlusion situation in both groups. It is concluded that the number of options generated depends on the strategies used to solve an action and the time available to visualize a given game situation.

KeYWORDS: Volleyball; Cognition; Decision-making; Options generation.

\section{Referências}

1. Arroyo MPM, Domínguez AM, Espá AU, González LG, Álvarez FDV. Representación de problemas tácticos en colocadoras de voleibol de las selecciones nacionales espańolas: efecto de la pericia. Rev Iberoam Psicol Ejerc Deport. 2008;3(2):229-40.

2. Vila-Maldonado S, Gallego NMS, Aberllán J, Jordán ORC. Efecto del tipo de colocación en el comportamiento visual y la toma de decisiones en bloqueadores de voleibol. Cult Cienc Deporte. 2012;7(20):103-14.

3. Vila-Maldonado S, Abellán J, Gallego NMS, López LMG, Jordán ORC. Decision-making and visual perception skills in youth volleyball players and non-players. J Sports Health Res. 2014;6(6):265-76.

4. Vila-Maldonado S, Sáez-Gallego NM, Abellán J, García-López LMG. Análisis de la toma de decisiones en la acción de bloqueo en voleibol: comparación entre jugadoras de élite y amateur. Rev Psicol Deport. 2014;23(2):239-46.

5. Raab M. Decision making in sport: influence of complexity on implicit and explicit learning. Int J Sport Exerc Psychol. 2003;1(4):406-33.

6. Raab M. SMART-ER: a Situation Model of Antecipated Response consequences in Tactical decisions in skill acquisition extended and revised. Front Psychol. 2014;5:1533.

7. Raab M. Think SMART, not hard - a review of teaching decision making in sport from an ecological rationality perspective. Phys Educ Sport Pedagogy. 2007;12(1):1-22.

8. Domínguez AM, Álvarez FDV, García-González L, Arias AG, Arroyo MPM. Intervención en la toma de decisiones em jugadores de voleibol em etapas de formación. Rev Psicol Deport. 2011;20(2):785-800.

9. Domínguez AM, Arroyo MPM, García-González L, Calvo TG, Álvarez FDV. Diferencias em la planificación de estrategias em voleibol entre jugadoras expertas y noveles. Apunts Educ Fis Deport. 2010;102:31-7.

10. Janelle CM, Hatfield BD. Visual attention and brain processes that underlie expert performance: implications for sport and military psychology. Mil Psychol. 2008;20(1):39-69.

11. Palmi J. La percepción: enfoque funcional de la visión. Apunts Educ Fis Deporte. 2007;88:81-5.

12. Williams AM, Grant A. Training perceptual skill in sport. Int J Sport Psychol. 1999;30(2):194-220.

13. Afonso J, Garganta J, Mesquita I. A tomada de decisão no desporto: o papel da atenção, da antecipação e da memória. Rev Bras Cineantropom Desempenho Hum. 2012;14(5):592-601.

14. Brandt R, Viana MS, Segato L, Andrade A. Atenção em velejadores: conceitos e aplicaçóes. Rev Bras Cien Esporte. 2012;34(1):69-80.

15. Hüttermann S, Memmert D. The influence of motivational and mood states on visual attention: a quantification of systematic differences and casual changes in subjects' focus of attention. Cogn Emot. 2015;29(3):471-83.

16. Hüttermann S, Memmert D. Does the inverted-U function disappear in expert athletes? An analysis of the attentional behavior under physical exercise of athletes and non-athletes. Physiol Behav. 2014;131:87-92.

17. Oliveira RF, Lobinger BH, Raab M. An adaptive toolbox approach to the route to expertise in sport. Front Psychol. $2014 ; 5: 709$.

18. Sanfey AG. Decision neuroscience: new directions in studies of judgment and decision making. Curr Dir Psychol Sci. 2007;16(3):151-5.

19. Johnson J, Raab M. Take the first: option-generation and resulting choices. Organ Behav Hum Decis Process. 2003;91(2):215-29.

20. Laborde S, Raab M. The tale of hearts and reason: the influence of mood on decision making. J Sport Exerc Psychol. 2013;35(4):339-57.

21. Raab M, Johnson JG. Expertise-based differences in search and option-generation strategies. J Exp Psychol Appl. 2007;13(3):158-70.

22. Furley P, Memmert D. Creativity and working memory capacity in sports: working memory capacity is not a limiting factor in creative decision making amongst skilled performers. Front Psychol. 2015;6:115. 
23. Memmert D. Creativity, expertise, and attention: exploring their development and their relationship. J Sports Sci. 2011;29(1):93-102.

24. Memmert D, Baker J, Bertsch C. Play and practice in the development of sport-specific creativity in team ball sports. High Abil Stud. 2010;21(1):3-18.

25. Memmert D, Roth K. The effects of non-specific and specific concepts on tactical creativity in team ball sports. J Sports Sci. 2007;25(12):1423-32.

26. Causer J, Ford PR. "Decisions, decisions, decisions": transfer and specificity of decision-making skill between sports. Cog Process. 2014;15(3):385-9.

27. Laborde S, Raab M, Kinrade NP. Is the ability to keep your mind sharp under pressure reflected in your heart? Evidence for the neurophysiological bases of decision reinvestment. Biol Psychol. 2014;100:34-42.

28. Raab M, Laborde S. When to blink and when to think: preference for intuitive decisions results in faster and better tactical choices. Res Q Exerc Sport. 2011;82(1):89-98.

29. Pizzera A, Raab M. Perceptual judgments of sports officials are influenced by their motor and visual experience. J Appl Sport Psychol. 2012;24(1):59-72.

30. Costa GCT, Castro HO, Cabral FA, Morales JCP, Greco PJ. Content validity of scenes of the declarative tactical knowledge test in volleyball - DTKT:Vb. Rev Bras Cineantropom Desempenho Hum. 2016;18(6):629-37.

31. Bordini FL, Costa MA, Medina-Papst J, Camata TV, Marques I. Efeito da oclusão temporal na ação de ataque sobre a tomada de decisão defensiva na modalidade de voleibol. Rev Bras Educ Fis Esporte. 2015;29(1):107-18.

32. Liu S. Research on athletes visual search: a case study. Int J Sci Res. 2015;4(1):1373-6.

33. Memmert D, Hüttermann S, Orliczek J. Decide like Lionel Messi! The impact of regulatory focus on divergent thinking in sports. J Appl Soc Psychol. 2013;43(10):2163-7.

34. Belling PK, Suss J, Ward P. The effect of time constraint on anticipation, decision making, and option generation in complex and dynamic environments. Cogn Technol Work. 2015;17(3):355-66.

35. Belling PK, Suss J, Ward P. Advancing theory and application of cognitive research in sport: using representative tasks to explain and predict skilled anticipation, decision-making, and option-generation behavior. Psychol Sport Exerc. 2015;16(1):45-59.

36. Kalis A, Kaiser S, Mojzisch A. Why we should talk about option generation in decision-making research. Front Psychol. 2013;4:555.

37. Memmert D, Perl J. Analysis and simulation of creativity learning by means of artificial neural networks. Hum Mov Sci. 2009;28(2):263-82.

38. Williams AM, Ford PR. Expertise and expert performance in sport. Int Rev Sport Exerc Psychol. 2008;1(1):4-18.

39. Williams AM, Reilly T. Talent identification and development in soccer. J Sports Sci. 2000;18(9):657-67.

40. Kreitz C, Furley P, Memmert D, Simons DJ. Working-memory performance is related to spatial breadth of attention. Psychol Res. 2015;79(6):1034-41

41. Laborde S, Furley P, Schempp C. The relationship between working memory, reinvestment, and heart rate variability. Physiol Behav. 2015;139:430-6.

42. Lola AC, Tzetzis G, Zetou H. The effect of implicit and explicit practice in the development of decision making in volleyball serving. Percept Mot Skills. 2012;114(2):665-78.

43. Moriya J, Koster EH, De Raedt R. The influence on work memory on visual search for emotional facial expressions. J Exp Psychol Hum Percept Perform. 2014;40(5):1874-90.

44. Afonso J, Mesquita I. Skill-based differences in visual search behaviours and verbal reports in a representative film-based task in volleyball. In J Perform Anal Sport. 2013;13:669-77.

45. Piras A, Lobietti R, Squatrito S. Response time, visual search strategy, and anticipatory skills in volleyball players. J Ophthalmol. 2014;189268:1-10.

46. Anderson JR. Acquisition of cognitive skill. Psychol Rev. 1982;89(4):369-406.

ENDEREÇO:

Henrique de Oliveira Castro

Av. Presidente Antônio Carlos, 6.627 31310-250 - Belo Horizonte - BRASIL e-mail: henriquecastro88@yahoo.com.br
Recebido para publicação: 03/o8/2015

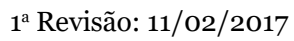

$2^{\text {a }}$ Revisão: 02/12/2017

Aceito: 20/12/2017 